\title{
Krise des Arbeitsrechts - Individualisierung der Anerkennungsverhältnisse?
}

Seit Längerem ist das deutsche Arbeitsrecht Schritten und Forderungen der Deregulierung ausgesetzt. Inzwischen werden seine Notwendigkeit und Zwecksetzung auch grundsätzlich infrage gestellt, so etwa mit der These, das Arbeitsverhältnis unterscheide sich nicht von anderen schuldrechtlichen Vertragsverhältnissen. Infolge grundlegender Veränderungen der Arbeitsbeziehungen könne nicht mehr generell von der Schutzbedürftigkeit des Arbeitnehmers oder von Interessengegensätzen die Rede sein. Dieser Aufsatz soll dazu beitragen, sich der grundlegenden Bedeutung des Arbeitsrechts neu zu vergewissern und den Wandel in seinen Auswirkungen auf das Arbeitsrecht zu betrachten. Dies geschieht in einer anerkennungssoziologischen Perspektive.

\section{Einleitung}

Das Arbeitsrecht befindet sich in einem steten Wandlungsprozess, der teilweise von Veränderungen in der Arbeitswelt angeregt wird, teilweise politischen Themenkonjunkturen und sozialen Auseinandersetzungen entstammt, teilweise paradigmatischen Wechseln in der Arbeitsrechtswissenschaft selbst geschuldet ist. Es gibt Anzeichen, dass sich derzeit Umbrüche andeuten, die es berechtigt erscheinen lassen, von einer sich abzeichnenden Krise des Arbeitsrechts zu sprechen. Denn gegenwärtig werden Grundlagen des Arbeitsrechts infrage gestellt und seine reale Wirkungskraft schwindet. Es ist auch deshalb von einer Krise zu sprechen, weil zwei Entwicklungen in der Arbeitswelt gleichzeitig hierfür verantwortlich gemacht werden können: Zum einen nämlich die Veränderung der Kräfteverhältnisse in den Arbeitsbeziehungen und auf dem Arbeitsmarkt, die vor dem Hintergrund vielfältiger Faktoren wie Globalisierung, Massenarbeitslosigkeit, neue Arbeitsformen, Tertiarisierung der Wirtschaft usw. zu erklären ist. Dies hat die tendenziell abnehmende Durchsetzungsmacht der Gewerkschaften zur Folge. Zum anderen aber ändern sich auch die Anerkennungserwartungen der Arbeitenden und die Normen, auf die bezogen Anerkennung gewährt oder vorenthalten wird. Damit verändert sich auch der normative Horizont, in dem Interessen definiert und artikuliert werden und deren Legitimation. Im Folgenden wird der Fokus der Betrachtung auf die Veränderung der Anerkennungsverhältnisse gelegt, um die Krise des Arbeitsrechts zu beschreiben.

\section{Arbeit und Anerkennung}

Arbeit ist nicht nur Gegenstand einer instrumentellen Tauschbeziehung, sondern in modernen Gesellschaften zugleich eine wesentliche Grundlage der Identität. Arbeitsplatz und Betrieb sind nicht nur Orte ökonomischen Tauschs bzw. von Ausbeutung. Die Arbeitswelt ist vielmehr Teil der Lebenswelt der Arbeitenden und durchzogen von Anerkennungsbeziehungen (Voswinkel 2005; Holtgrewe et al. 2000). Allerdings bedeutet dies keineswegs, dass Anerkennung der Zweck des Betriebes wäre. Sie wird auch dann, wenn dem Management an einem guten Betriebsklima und einem humanen Umgang mit den Beschäftigten gelegen ist, nur unter dem Vorbehalt gewährt, dass sich die Arbeitsbeziehung „rechnet" (Kotthoff 2000).

Dass die Arbeit zu einem solch zentralen Bezugspunkt von Anerkennung und Identität wurde, ist von der Geschichte der Arbeit her gesehen alles andere als selbstverständlich: Erst in der Moderne wird das Leistungsprinzip in der Arbeit zentrales Element des Selbstverständnisses und der Rechtfertigungsordnung der Gesellschaft. Dass Anerkennung auf Arbeit gegründet sein möge, richtete sich als normativer Anspruch historisch gegen die Privilegien des Adels und der ständischen Gesellschaft. Die Arbeiterbewegung wendete diesen Anspruch gegen das Bürgertum: Die Arbeiter sind es, die die Werte schaffen! Gerade in und um die Arbeit findet somit ein Kampf um Anerkennung statt, der auch scheinbar „nur" instrumentellen Auseinandersetzungen, etwa um das Entgelt, ihre moralische Dynamik verleiht.
Arbeit und Betrieb sind somit allerdings auch ein Terrain von Missachtungserfahrungen. Denn wenn sich Anerkennungserwartungen auf die Arbeit beziehen, geht dies auch mit der Erfahrung verfehlter und vorenthaltener Anerkennung einher. Nicht nur die Arbeitslosen erleben dies durch ihren Ausschluss von der Anerkennungsquelle Arbeit, sondern auch diejenigen, deren Leistung nur gering geschätzt wird.

Wichtig dafür, dass die Arbeit überhaupt Quelle von Anerkennung werden konnte, war die Befreiung der Arbeit aus Formen der Leibeigenschaft und der ständischen Einhegung. Zwang und Arbeit wurden im Übergang zur kapitalistischen Moderne prinzipiell entkoppelt (Castel 2000, S. 152ff.). Hierfür steht die Institution des freien Arbeitsvertrags ${ }^{1}$, die prinzipielle normative Erhebung der Arbeitenden zu freien und gleichen Bürgern und Rechtssubjekten. Anders als in der Ständegesellschaft sind rechtliche Anerkennung und soziale Wertschätzung fortan nicht mehr aneinander geknüpft. Die Rechte der Einzelnen sind nicht mehr von ihrer Standeszugehörigkeit abhängig und grundsätzlich steht allen ein Recht auf Entfaltung ihrer Persönlichkeit zu. Sozialer Aufstieg ist durch Arbeit möglich und soll auf Leistung beruhen.

\footnotetext{
1 Im juristischen Sinne handelt es sich um den Dienstvertrag nach § 611 BGB.
}

Stephan Voswinkel, Dr. habil., ist Soziologe am Institut für Sozialforschung Frankfurt a. M. Arbeitsschwerpunkte: Arbeits- und Wirtschaftssoziologie. e-mail: voswinkel@em.uni-frankfurt.de 
Die soziale Realität zeigte im frühen Kapitalismus jedoch ein anderes Gesicht. Der prinzipiell freie Arbeiter war nicht nur arm, sondern besaß auch nach wie vor ein nur geringes Ansehen. Gegenüber den Zunftverhältnissen, die ihm einen gewissen sozialen Status verliehen, war sein Sozialprestige sogar noch gesunken. Die rechtliche Freiheit erwies sich als formale. Auch auf politischer Ebene bestanden Diskriminierungen, etwa durch das Klassenwahlrecht, lange Zeit fort. Vor allem stellte sich die Arbeitswelt als eingekapselte Zone in der bürgerlichen Welt dar, als faktisch „rechtsfreier Raum“, in dem die alte Welt nicht rechtlich, aber sozial faktisch fortlebte, günstigenfalls humanisiert durch Relikte patriarchalischer Fürsorglichkeit. Dieser innere Widerspruch der freien Lohnarbeit bedurfte einer Lösung: als Antwort auf einen zähen Prozess von Klassenauseinandersetzungen, der sich auch als Kampf um Anerkennung (Honneth 1994) beschreiben lässt.

\section{Arbeitsrecht als Institutionalisierung von Anerkennungsverhältnissen}

Ein Beitrag zu dieser Lösung war das sich entwickelnde Arbeitsrecht. Es reagierte auf die der Arbeitsbeziehung unangemessene Form des individuellen freien Arbeitsvertrags, der den Tausch von Arbeitskraft gegen Entgelt als einen normalen Warentausch behandelt, für den dementsprechend die allgemeinen schuldrechtlichen Regeln gelten. Dies entsprach der Befreiung der Arbeit aus den hergebrachten ständisch-feudalen Fesseln, die H. S. Maine (1997) Mitte des 19. Jahrhunderts auf die Formel "from status to contract" brachte. Der freie Arbeitsvertrag ignoriert damit jedoch dreierlei:

(1) Erstens ist die Arbeitskraft keine Ware wie jede andere. Sie ist nicht von der Person des Arbeitenden zu trennen. Vielmehr vermietet der Arbeitende immer auch seine Person mit, weil er seine Arbeitskraft nicht einfach am Werkstor zum Arbeiten abgeben kann.

(2) Zweitens ist der Arbeitsvertrag ein unvollständiger Vertrag. Er regelt den zeitlichen Umfang der Vermietung der Arbeits- kraft und die Höhe des Entgelts, nicht aber den genauen Inhalt, den Grad und die Qualität der geschuldeten Leistung. Anders als ein Werkvertrag bestimmt er kein bestimmtes Ergebnis, sondern die Zurverfügungstellung der Arbeitskraft für einen Zeitraum. Genaueres bleibt dann dem Direktionsrecht des Arbeitgebers überlassen. Ein Arbeitsvertrag bestimmt somit die formal freiwillige zeitlich begrenzte Außerkraftsetzung des Selbstbestimmungsrechts des Beschäftigten. Diese Verbindung von Freiheit und Unfreiheit steht jedoch im Kontrast zum Subjektverständnis der Moderne und zur rechtlichen Anerkennung aller Subjekte der Gesellschaft als freie Personen.

(3) Drittens findet für die meisten Beschäftigten der Abschluss eines Arbeitsvertrags auf der Basis ungleicher Machtverhältnisse auf dem Arbeitsmarkt statt, weil der Arbeitskraftbesitzer seine Ware nur in sehr engem Rahmen zurückhalten kann und deshalb auch bei sinkenden Entgelten Arbeitsgelegenheiten nachfragen muss.

Das Arbeitsrecht hat im Kern also drei Aufgaben:

- Es soll die Ausübung des Direktionsrechts regulieren;

- es soll die Menschenwürde der Arbeitenden sichern und ihre rechtliche Anerkennung auch im Betrieb gewährleisten;

- es soll durch die Verringerung der Asymmetrie der Machtverhältnisse auf dem Arbeitsmarkt die sozialen Voraussetzungen für die Wahrnehmung der Rechte vertragsfähiger Bürger schaffen; hierfür steht insbesondere die Institution der Tarifautonomie.

Diese Aufgaben kann das Arbeitsrecht nur erfüllen, wenn es entgegen der Individualität des Arbeitsvertrags typisiert und kollektiviert. Es etabliert generalisierte Regeln, an denen sich Arbeitsverträge und die Ausübung des Direktionsrechts orientieren müssen, und es stützt auf der Basis der Koalitionsfreiheit mit dem Tarifvertragsrecht die kollektive Vertragsaushandlung. In Deutschland etablierte sich mit der betrieblichen und der Unternehmensmitbestimmung eine spezifische Konstitutionalisierung der Organisation. Durch diese Formen kollektiver Interessenvertretung wird die Arbeit als kollektive Kategorie anerkannt. Und auf der Basis dieser kollektiven und rechtlichen Anerkennung konnte sich der Status der Lohnarbeit entwickeln, den T. H.Marshall (1992/1950) als ,industrial citizenship“ bezeichnet hat. Auf diese Weise folgte auf die Bewegung „vom Status zum Vertrag“ die Gegenbewegung „vom Kontrakt zum Status“ (Castel 2000). Sie war allerdings doppelgesichtig: Sie verband nämlich die bürgerschaftliche Anerkennung der Arbeitenden mit ihrer Missachtung in tayloristischen Arbeitsstrukturen, in ihrer Reduktion auf funktional eingepasste Ausführende in einem vorab geplanten effektivierten Funktionssystem. In dieser spezifischen Kombination von Anerkennung und Missachtung kann sie als „fordistische“ Institutionalisierung von Anerkennung bezeichnet werden.

Industrial citizenship, Arbeitsrecht und Sozialstaat bildeten zugleich die Grundlage für den Prozess der Individualisierung (Leisering 1998). Nunmehr konnte sich die Vorstellung verallgemeinern, eigene biografische Projekte verfolgen, sozialen Aufstieg für sich oder die Kinder ermöglichen zu können und zu dürfen. Soziale Wertschätzung wurde nun stärker als Individuum angestrebt bzw. dem Individuum zugewiesen. In der Begrifflichkeit von Boltanski und Chiapello (2003) kann man sagen, dass der relative Erfolg der ,sozialen Kritik“, der sich gerade auch in der Entwicklung eines Arbeitsrechts zeigte, die Voraussetzungen für die Ausprägung einer „künstlerischen“2 Kritik schuf, der es um mehr Autonomie und Selbstbestimmung in der Arbeit ging und die in Deutschland unter anderem in der Zielsetzung einer Humanisierung und im normativen Anspruch einer Subjektivierung der Arbeit (Baethge 1991) zum Ausdruck kam.

„Künstlerisch“ war auch die Kritik an der Verrechtlichung als einer „Kolonialisierung" der Lebenswelt: Die Subjekte seien gezwungen, ihre Lebensweise und -pläne und den Umgang mit Konflikten in das vom Raster des Rechts vorgegebene Korsett

\footnotetext{
Boltanski und Chiapello identifizieren zwei Formen der Kritik am Kapitalismus, die eine unterschiedliche Stoßrichtung entwickeln und miteinander in Konflikt geraten können: Die "soziale Kritik" richtet sich gegen soziale Ungleichheit und Unsicherheit, die "künstlerische Kritik" gegen die Einschränkung autonomer und selbstbestimmter Handlungsmöglichkeiten. Letztere trat Ende der 1960er Jahre in den Vordergrund und wurde insbesondere in der Abkehr vom tayloristischen Leitbild wirksam.
} 
einzupassen, um den entsprechenden Schutz und Status zu erlangen (Habermas 1988, S. 530ff.). So wurden auch arbeitsrechtliche und sozialstaatliche Regulierungen als Gefährdungen der Autonomie verstanden. Insofern die Erfolge der sozialen Kritik erst die künstlerische ermöglicht hatten, wendete sich die künstlerische Kritik gegen ihre eigenen Voraussetzungen. Ansprüche auf Selbstverwirklichung in der Arbeit standen dem traditionellen Pflichtethos gegenüber. Schutzrechte sollten nicht mehr auf der Abhängigkeit des Arbeitnehmers beruhen, und viele Beschäftigte begriffen sich weniger als Teil eines Kollektivs denn als Individuum. Auf diese Weise verloren sowohl vorherrschende Formen der Anerkennung als auch Grundparadigmen des Arbeitsrechts an Akzeptanz. Und dies in einer Phase, als gerade erst im Arbeitsrecht ein Paradigmawechsel - mit erheblichem Zeitverzug zur gesellschaftlichen Entwicklung-erfolgreich vollzogen schien, der als Abkehr vom Patriarchalismus bezeichnet werden kann.

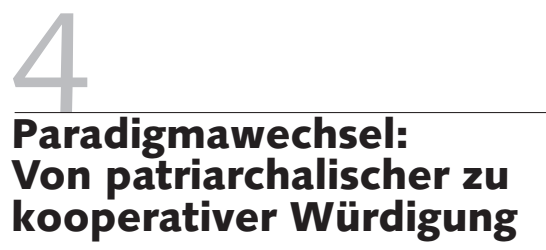

In der Geschichte des deutschen Arbeitsrechts lassen sich zwei Paradigmen, also konzeptionelle Muster, unterscheiden, mit denen es versuchte, den Besonderheiten des Arbeitsvertrags gerecht zu werden. Beiden gemeinsam ist der Bezug auf die Kollektivität der Arbeit und auf den Betrieb. Sie institutionalisieren eine Form der Anerkennung, die ich als Würdigung bezeichne (Voswinkel 2001, S. 281ff.). Sie bezieht sich auf Zugehörigkeit (hier zum Betrieb), gewürdigt werden der Beitrag zu einem gemeinsamen Leistungsergebnis, die Einordnung in den Betrieb und die Akzeptanz des Direktionsrechts sowie die Orientierung der Lebensplanung auf das Unternehmen. In dieser Anerkennungsform wird das Arbeitsverhältnis als eine auf Dauer und wechselseitige Bindung angelegte Beziehung sozialen Tauschs behandelt. Würdigung zeigt sich hier, wie Kotthoff (2000, S. 31) im Anschluss an Gouldner gezeigt hat, vor allem in Formen der Rücksichtnahme: Bei Fehlern soll man eine zweite Chance erhalten, familiäre Verpflichtungen dürfen nicht gänzlich außer Acht gelassen werden, wer am Längsten da ist, soll besonders geachtet werden, auf ältere und leistungsgeminderte Arbeitnehmer sollte Rücksicht genommen werden.

Die beiden Muster des Arbeitsrechts konzipieren jedoch das Verhältnis von Arbeit und Kapital und die Sozialverfassung des Betriebs sehr unterschiedlich. Sie formen die Anerkennung

- im einen Falle als patriarchalische Würdigung in einer Betriebsgemeinschaft und - im anderen Falle als kooperative Würdigung auf Basis der Anerkennung als Arbeitsbürger.

Die patriarchalische Würdigung ist gewissermaßen eine Anerkennung „von oben nach unten“. Im Arbeitsrecht kam sie prägnant in der Lehre vom „personenrechtlichen Gemeinschaftsverhältnis“ zum Ausdruck, die noch in den 1950er und 60er Jahren etwa in den einflussreichen Lehrbüchern von Nikisch und Hueck/Nipperdey lebendig blieb ${ }^{3}$ (Annuß 2004, S. 297ff.). Ihr liegt eine hierarchische Vorstellung von Gemeinschaft zugrunde, die jedoch durch die Würdigung der Leistungen und der „Treue“ der Beschäftigten ${ }^{4}$ auf der einen Seite und durch die Vorstellung einer sozialen Verantwortung der Arbeitgeber auf der anderen Seite eine ,vertikale“ Anerkennungsform „harmonischer Ungleichheit" (Wagner 2004, S. 172ff.) enthielt.

Die kooperative Würdigung dagegen versteht die Arbeitenden als autonome, in ihrer Kollektivität gleichwertige Partner des Unternehmens. Diese Anerkennung wird institutionalisiert in Tarifverträgen, und in Deutschland auch in der Mitbestimmung. Beide Rechtsinstitutionen symbolisieren, dass die Reduktion von Würdigung auf Fürsorge abgelehnt wird. Dem Arbeitsrecht kommt die Bedeutung zu, die Anerkennung in einen Rechtsanspruch zu fassen, den Anspruch des rechtlich geachteten Arbeitsbürgers (Kotthoff 1994, S. 334). Die intermediäre Regulierungsebene des Tarifvertrags und der Mitbestimmung ist die Voraussetzung für eine relativ freie Gestaltung der Individualarbeitsverträge - auch für die Beschäftigten. Die arbeitsrechtlichen Regeln können ,in dem Maße Statusrechte des einzelnen Beschäftigten begründen, wie sie gegenüber temporalen, sektoralen und lokalen Schwankungen der Arbeits- und insbesondere der Produktmärkte unempfindlich gemacht werden" (Streeck 1988, S. 25). Weil diese Anerken- nung der Arbeit im Arbeitsrecht allerdings verbunden war mit ihrer Missachtung in der tayloristischen Arbeitsform, spitzten sich die Spannungen in den fordistischen Anerkennungsverhältnissen zu.

\section{Erosion der "fordistischen" Anerkennungsverhältnisse}

Die beginnende Abkehr von tayloristischen Arbeitsformen war allerdings noch keine Problemlösung. Eher gab es eine Problemverschiebung: Zwar nahm der Anteil an Arbeitsformen ab, die den arbeitenden Menschen, seine Subjektivität missachteten, jedoch erodierten zugleich die strukturellen Grundlagen der fordistischen Anerkennungsverhältnisse, ohne dass etwas anderes an ihre Stelle trat:

(1) Unternehmensstrukturen dezentralisieren sich, Organisationshandeln wird unmittelbarer an den Markt gekoppelt, sodass sich Unternehmen flexibilisieren. Die Marktanforderungen werden in Form kurzfristigerer Beschäftigungshorizonte an die Beschäftigten weitergereicht. Outsourcing führt zur Ausdifferenzierung von Organisationen und zur Rekombination in Netzwerkstrukturen. Dies geht einher mit der Aufwertung kurzfristiger Erfolgsmaßstäbe, von Kurzfrist-Ökonomie und Kurzfrist-Bindung (Moldaschl/Sauer 2000; Schumann 1998).

(2) Das Leistungsverständnis verändert sich hin zu einer Ergebnisorientierung, die Leistungsaufwand, eingebrachte Qualifikationen und Anstrengung in ihrer Wertschätzung und finanziellen Honorierung unter den Vorbehalt des erreichten Ergebnisses, insbesondere des Erfolgs am Markt stellt (Voswinkel 2003; Bender 1997). An die Stelle von Verfahrensvorgaben tritt die Ergebnisbewertung; Arbeitsaufwand und aufgebrachte Arbeitszeit werden für die Bewertung der Leistung irrelevant.

\footnotetext{
3 Eine extrem autoritäre Form erhielt es in der Zeit des Nationalsozialismus im Gesetz zur Ordnung der Nationalen Arbeit (AOG) im Jahre 1934.

4 Die Beschäftigten besaßen wiederum auch eine "Treuepflicht".
} 
(3) Zunehmend entwickeln mehr Arbeitende Ansprüche an die Arbeit: sich selbst verwirklichen, Arbeit selbst organisieren zu können oder in ihr gar "Spaß" zu haben (Pongratz/Voß 2003). Zugleich wird dies als Erwartung an sie herangetragen („Subjektivierung der Arbeit") (Moldaschl/Voß 2002). Dem neuen Leitbild entsprechend soll sich ein moderner Arbeitnehmer als Unternehmer seiner selbst verhalten. In den Unternehmen herrscht ein entsprechender Inszenierungsanreiz, der es illegitim erscheinen lässt, im Sinne des fordistischen Arbeitnehmers pflichtgemäß seine Arbeit zu tun und auf Anweisungen zu warten (Kocyba 2000). Einem solchen Identitätsideal entspricht es nicht, sich als schutzbedürftiger Arbeitnehmer zu verstehen. Auch wird demjenigen, der letztlich „für sich" arbeitet, die sozialmoralische Basis entzogen, Würdigung seiner Arbeit fürs Unternehmen $\mathrm{zu}$ erwarten (Voswinkel 2002).

(4) Nicht nur die „Subjektivierung der Arbeit" lässt Arbeit als ein erstrebenswertes Gut erscheinen. Vielmehr sorgen die Knappheitsverhältnisse auf den Arbeitsmärkten dafür, dass Arbeit nicht mehr nur das ist, wofür man be-lohnt wird, sondern selbst Belohnung ist - das Honorar erfolgreicher Arbeitsplatzsuche, der richtigen mentalen Einstellung, der Sorge um die employability. Arbeit wird zur Probe für die Arbeit: Befristete Arbeitsverhältnisse, Phasen von Praktika und Traineeprogramme etablieren Arbeit als Bewährungszeit, in der man sich immer erneut um weitere Arbeit bewirbt.

Diese Entwicklungen lassen sich nun in der Perspektive der Anerkennung so zusammenfassen, dass Arbeit kaum mehr als Aufwand, als Opfer des Arbeitenden wahrgenommen wird. Folglich darf er nicht Anerkennung als Gegenleistung erwarten. Arbeit an sich ist bereits Belohnung: Arbeits-Erwerb als Anerkennung „des Arbeitsmarkts" und Zeichen gesellschaftlicher Zugehörigkeit, Arbeit als Chance der Selbstentfaltung. Nicht mehr für Erfahrungen und erbrachte Leistungen kann man Anerkennung verlangen, sondern es geht um Potenziale für die Zukunft, die von vergangenen Leistungen eher gehemmt werden. Anerkennung erfährt man nun eher für den individuellen, vor allem ökonomischen Erfolg. Sie ist jedoch fragil und muss stets neu erworben werden. Erworbener
Status wird delegitimiert, Bande langfristiger Reziprozität werden zerschnitten (Voswinkel 2005, S. 252f.). Bindungen entstehen nun eher durch wechselseitige Versprechungen zukünftiger Win-Win-Konstellationen und sind stets vorbehaltlich und reversibel. Sie entsprechen einem neuen Übergang vom Status zum Vertrag (Streeck 1988). Weil so institutionell verankerte Formen der Anerkennung entfallen, wird das Bedürfnis nach Anerkennung drängender, werden Kämpfe um Anerkennung individueller geführt.

\section{Aktueller Wandel des Arbeitsrechts: Vom Status zum Vertrag?}

Damit werden die veränderten Bindungsgrundlagen für das Arbeitsrecht bedeutsam, das ja gerade den Arbeitsvertrag mit modernen Statuselementen unterlegt hat. Allerdings setzen sich Veränderungen in den Arbeitsmarkt- und Beschäftigungsverhältnissen nicht einfach in arbeitsrechtliche Veränderungen um. Das Arbeitsrecht folgt einer eigenen Logik, neue Regelungen folgen einem Anschlusszwang an bestehende (Luhmann 1981, S. 87). Die Arbeitsrechtler bilden eine eigene Professionskultur mit untereinander keineswegs einheitlichen, aber doch speziellen Argumentationsmustern und Leitbildern aus (Mückenberger/Supiot 2000, S. 117ff.), in denen Veränderungsanforderungen der Arbeitswelt reformuliert werden. In den hierüber geführten eher grundlagentheoretischen Debatten in der Arbeitsrechtsprofession sind die konkreten arbeitspolitischen Bezüge oft nur indirekt erkennbar. Gleichwohl kann man einige paradigmatisch relevante Themen hervorheben, die für den Wandel des Arbeitsrechts bedeutsam sind:

\subsection{ABKEHR VOM FÜRSORGE- GEDANKEN}

Obwohl die Fürsorgesemantik im Arbeitsrecht bereits zuvor zurückgedrängt worden war, wird doch erneut die Abkehr von „patriarchalischen“ oder „paternalistischen“ Elementen des Arbeitsrechts gefordert. Eine eher semantische Anpassung findet etwa im Arbeitsrechtshandbuch von Schaub statt, der dafür plädiert, auf den Begriff der
„Fürsorgepflicht“ zugunsten der „Schutzpflicht" zu verzichten (Schaub 2005, S. 1067). Weiter gehen Autoren, die den Schutzcharakter des Arbeitsrechts generell als paternalistische Überformung der Vertragsfreiheit charakterisieren (etwa Schwarze 2005). Schlichtere Argumentationen behaupten einfach die Marktwidrigkeit der Arbeitsrechtsentwicklung und kritisieren die Dysfunktionalität des Arbeitnehmerschutzes (Picker 2005).

Als ein Beispiel einer arbeitsrechtlichen Regulierung, die nur vor dem Hintergrund einer auf Dauer angelegten Arbeitsbeziehung begründbar ist, will ich die Entgeltfortzahlung im Krankheitsfalle anführen. Honoriert wird hier nämlich nicht die konkrete Arbeitsleistung, sondern die langfristige Vermietung ${ }^{5}$ der Arbeitskraft. Erkrankt der Beschäftigte, so leistet er zwar aktuell nichts, aber er bleibt doch Mitglied der Organisation, Teil einer sozialen Beziehung. Deshalb steht ihm die Fortzahlung des Entgelts zu; anders in einem Paradigma, in dem der Beschäftigte als Unternehmer seiner Arbeitskraft konzipiert wird. Von diesem darf man erwarten, dass er mit seiner Arbeitskraft ökonomisch, unternehmerisch umgeht und sie zu erhalten weiß. Wird er krank, ist ihm dies nicht gelungen und er leistet - in der kurzfristigen Perspektive der Vertragserfüllung - nicht, weshalb eine Entgeltfortzahlung in der Logik des Leitbildes „Arbeitskraftunternehmer“ unverständlich bliebe.

\subsection{TYPISIERUNG ALS PROBLEM}

Das Arbeitsrecht bezieht sich auf Statuselemente, indem es typisiert. So bindet es beispielsweise die Unterlegenheit des Arbeitenden gegenüber dem Arbeitgeber auf dem Arbeitsmarkt und seine Weisungsgebundenheit an die Arbeitnehmereigenschaft. Auch das Kündigungsrecht arbeitet etwa bei der sozialen Auswahl mit Typisierungen wie Lebensalter, Betriebszugehörigkeit, Versorgungspflichten. Die soziologische Kategorie des Normalarbeitsvertrages ist eine Kombination verschiedener Typisierungen: auf unbestimmte Zeit geschlossener Arbeitsvertrag, Vollzeitarbeit,

\footnotetext{
5 Dies ist nicht juristisch gemeint. Der Terminus "Vermietung" soll vielmehr darauf hinweisen, dass der bekanntere des "Verkaufs" der Arbeitskraft unkorrekt ist, weil die Arbeitskraft nicht ein für allemal verkauft wird.
} 
Weisungsabhängigkeit. Ein Blick auf reale Differenzen von Beschäftigungsstabilität zeigt jedoch, dass unbefristete Arbeitsverträge Beschäftigungsverhältnisse von sehr unterschiedlicher Stabilität regulieren und dass etwa ein befristeter Arbeitsvertrag zur Probe bei einem expandierenden Großunternehmen mehr Beschäftigungsperspektiven eröffnen kann als ein unbefristeter Arbeitsvertrag in einem kleinen Handwerksbetrieb. Eine Teilzeitarbeit kann mehr Entgelt einbringen als eine Vollzeitarbeit im Niedriglohnbereich. Die Typisierung des Arbeitsrechts kann also den realen Differenzen nie völlig gerecht werden.

Dieses Problem wird virulenter, wenn sich die Arbeits- und Lebensbedingungen der Arbeitenden zunehmend ausdifferenzieren, ja individualisieren. Typisierungen erscheinen dann als Schematisierungen, die der realen Lebens- und Interessenlage nicht gerecht werden. Deshalb, so Simitis schon 1984, müsse es darum gehen, den Verrechtlichungsprozess dort neu zu gestalten, wo „die regulierende Intervention (...) den Schutz des Arbeitnehmers nicht auch und gerade als Aufgabe versteht, seine individuelle Entscheidungskompetenz herzustellen und zu festigen" (Simitis 1984, S. 121). Andererseits sind Typisierungen unumgänglich, um der Vielfalt spezifischer Konstellationen Regeln zu setzen. Prozeduralisierungen können eine Alternative zu einem hohen Maß inhaltlicher Typisierungen sein, doch auch sie sind gebunden an Typisierungen von Verfahren und Interessenakteuren.

\subsection{NEUDEFINITION DES GÜNSTIGKEITSPRINZIPS}

Im Tarifvertrag werden kollektive Regelungen getroffen, die im Normalfall als Basisstandard fungieren sollen. Die Flexibilität soll dann durch übertarifliche Arbeits- und Entlohnungsbedingungen ermöglicht werden. Dabei soll das Günstigkeitsprinzip gewährleisten, dass vom Tarifvertrag nur $z u$ gunsten des Arbeitnehmers abgewichen werden kann. Lange Zeit war dieses Modell unbestritten und unproblematisch. Seit den 1990er Jahren jedoch ändert sich die inhaltliche Füllung des „Günstigkeitsprinzips“. Denn viele Arbeitsrechtler gehen davon aus, dass in den Günstigkeitsvergleich die Sicherung des Arbeitsplatzes einzubeziehen sei (Schaub 2005, S. 1966ff.; kritische Darstellung solcher Positionen in Höland et al. 2000). Mit dieser Argumenta- tionsfigur wird auch eine Abweichung von den tariflichen Arbeits- und Entgeltbedingungen nach unten legitimiert, wenn dies verspricht, zur Sicherung des Arbeitsplatzes beizutragen. Das stellt die durch den Tarifvertrag begründeten Standards letztlich unter den Vorbehalt der relativen Marktposition des einzelnen Arbeitnehmers bzw. des einzelnen Betriebs. In diesem veränderten Verständnis des Günstigkeitsprinzips wird deutlich, dass die Arbeit von einem zu honorierenden Aufwand zu einem erstrebenswerten Gut geworden ist, für das man Opfer bringt. Das kennzeichnet den Wandel vom Status zum Vertrag.

\subsection{NEUE FORMEN VON BESCHÄFTIGUNGSSTATUS UND LEISTUNGSREGULIERUNG}

Eine zentrale Typisierung des Arbeitsrechts besteht darin, dass die schutzbegründende Abhängigkeit an die Arbeitnehmereigenschaft gebunden ist und dass formale Selbstständigkeit für Selbstbestimmtheit steht. Diese Typisierung wird von zwei Seiten in Frage gestellt:

(1) Auf der einen Seite nehmen diejenigen abhängigen und auch faktisch weisungsgebundenen Beschäftigungen $\mathrm{zu}$, die rechtlich von Selbstständigen ausgeübt werden (Gerlmaier/Kastner 2003; Supiot 2001, S.3ff.). Das an die Kategorie des klassischen Arbeitnehmers gebundene Arbeitsrecht wird dadurch in seiner Reichweite immer weiter eingeschränkt. Lösungen, die das Arbeitsrecht bisher bereitstellt, sind die Einbeziehung der abhängig Selbstständigen als „arbeitnehmerähnliche Personen“ sowie der Ausbau und die Anwendung der entsprechenden Regelungen.

(2) Auf der anderen Seite verändert sich die Leistungsregulierung der abhängigen Arbeitnehmer, weil sich im rechtlichen Rahmen des Arbeitsvertrags ergebnisbezogene Regulierungs- und Vertragsformen durchsetzen, die den Beschäftigten scheinbar jede Freiheit bei der Ausführung der Arbeit und der Erreichung des vereinbarten Ergebnisses lassen, das allerdings recht präzise vereinbart bzw. über Kennziffern gesteuert wird. Viele Beschäftigte erfahren diese Regulierungsweise tatsächlich als einen Zugewinn an Autonomie, aus der jedoch zugleich ein großer Druck zur Selbstrationalisierung und -disziplinierung entsteht, um das Ziel auch zu erreichen (Peters
2003). Geschützt werden muss der Arbeitnehmer hier gewissermaßen vor sich selbst. Und reguliert werden müssen die Arbeitsformen wie auch die Arbeits- und Zeitorganisation selbst, die Anlass zur Selbstausbeutung und teilweise auch zum Raubbau an der Gesundheit geben (Kocyba/Voswinkel 2007). Zwar wird ein Arbeitsvertrag rechtlich nicht zum Werkvertrag, wenn in einem bestehenden Arbeitsvertrag auf Ergebnisorientierung umgestellt wird (Trittin 2003), doch kann man faktisch von hybriden Kombinationen von Arbeits- und Werkvertrag (Schmid 2002) sprechen, wenn Leistungen im Sinne von Ergebnissen und Zielen vereinbart und in Vertragsform gegossen werden.

Für das Arbeitsrecht stellt sich angesichts dieser Entwicklungen die hochproblematische Frage, wie Regulierungen aussehen können, die auf der einen Seite dem Selbstbestimmungsbedürfnis der Beschäftigten gerecht werden, die aber angesichts der Autonomieillusionen der Beschäftigten und der "Sachzwänge“, denen sie sich ausgesetzt sehen, auch nicht wirkungslos bleiben.

\subsection{REVERSIBILITÄT VON EINSTELLUNGEN}

Entscheidungen über die Zugehörigkeit zu einer Organisation bzw. zu einem Netzwerk betreffen vermehrt den Zugang. $\mathrm{Zu}$ Zeiten der Normalität des „Normalarbeitsverhältnisses" waren Kündigungen - nach einmal vorgenommener, auf Dauer angelegter Einstellung - die zentralen Entscheidungen über die Zugehörigkeit. Zwar spielen Kündigungen auch weiterhin eine wesentliche Rolle beim Zugang in Arbeitslosigkeit; der Bestandsschutz von Arbeitsverhältnissen hat also weiterhin große Bedeutung. Doch zunehmend werden nun die Einstellungs- und Zugangsentscheidungen reversibel gehalten, oder genauer gesagt: in ihrem Entscheidungsgehalt gemindert. Ein befristeter Arbeitsvertrag etwa wird nicht beendet, er endet automatisch, ohne Entscheidung. Wird er verlängert, so ist eine neue Zugangsentscheidung notwendig. Wer als Freelancer von Aufträgen abhängt, dem werden keine Aufträge entzogen, er muss sich stets um neue bemühen. Diese Umstellung von Ausschluss- auf Zugangsentscheidungen verändert die Legitimationserfordernisse und -mechanismen. Eine Kündigung ist nach rechtlichen und betrieblichen Regelungen 
begründungspflichtig; nicht aber die Auftragsvergabe an andere Auftragnehmer oder die Nicht-Verlängerung bzw. NichtErneuerung eines Arbeitsvertrages. Man kann hier von einer Umkehr der Begründungslast sprechen. Ein Bewerber hat plausibel zu machen, warum er der Richtige ist, im Falle der Kündigung muss hingegen der Arbeitgeber legitimieren, warum das Arbeitsverhältnis nicht fortgesetzt werden soll. Diese Umstellung vermindert faktisch die Reichweite des Arbeitsrechts, weil die Schutz- und Partizipationsrechte unter den Vorbehalt der allein beim Arbeitgeber bleibenden Entscheidung über die Fortsetzung der Vertrags- und Geschäftsbeziehung gestellt werden.

Das Arbeitsrecht bedarf einer weitreichenden Reform, will es diesem Wandel der Legitimationsmechanismen gerecht werden. Zentral ist eine solche Reform, weil die Begründungslastumkehr die Umstellung von einem klassischen Lohnarbeitsverhältnis auf Formen der flexiblen Netzwerkbeschäftigung reflektiert. Es gibt bislang eine Reform, die den Erfordernissen einer Zugangsregulierung gerecht werden soll: Das umstrittene Allgemeine Gleichbehandlungsgesetz (AGG). Es soll die Grund- und Menschenrechte auch in der Arbeitswelt in neuer Weise ins Spiel bringen, indem auch Entscheidungen über Einstellungen so getroffen werden sollen, dass sie nicht im Hinblick auf ethnische Herkunft, Geschlecht, Religionszugehörigkeit usw. diskriminierend sind. Die gegen das Gesetz erhobenen Einwände, die sich weniger gegen die Ziele als gegen Verfahren, Formalität, Schematismus und Missbrauchsmöglichkeiten wenden, zeigen zugleich, wie schwierig Regulierungen von Zugängen auszugestalten und zu implementieren sind. $\mathrm{Zu}$ gangsregulierungen müssten sich über die Abwehr von Diskriminierungen hinaus aber auch darauf richten, Kontinuität in Arbeits- und Auftragsbeziehungen zu fördern. ${ }^{6}$

\section{Resümee}

In der modernen Gesellschaft ist Arbeit ein wesentlicher Bezug der Anerkennung der Menschen. Indem sich hier die Anerken- nungsform des Rechts von derjenigen sozialer Wertschätzung entkoppelt, erhalten alle Individuen einen Anspruch auf rechtliche Anerkennung unabhängig von ihrem Status in der Gesellschaft. Der individuelle Arbeitsvertrag impliziert allerdings die Anerkennung des Direktionsrechts des Arbeitgebers und damit die Gefahr, dass Arbeitende „freiwillig“ vertraglich auf ihre grundlegenden Rechte (zeitweilig) verzichten. Um die Anerkennung als gleiche Subjekte auch in der Arbeitswelt zu gewährleisten, haben sich verschiedene Institutionen entwickelt. Zu diesen zählt das Arbeitsrecht, das dem Direktionsrecht Grenzen setzt, durch kollektive Aushandlungsinstitutionen eine Angleichung der Wettbewerbsbedingungen fördert und die betrieblichen Herrschaftsbeziehungen in eine konstitutionelle Anerkennungsordnung („industrial citizenship“) transformiert.

Nachdem patriarchalische Elemente zunehmend zurückgedrängt waren, gerät das Arbeitsrecht in neuerer Zeit durch Veränderungen der Arbeitswelt in eine Krise. Die Unternehmen werden instabiler, die Planungshorizonte verkürzt, die Beschäftigungsverhältnisse sind weniger auf Dauer angelegt. Langfristige generalisierte Reziprozitätsbeziehungen lösen sich auf, der individuelle Arbeitsvertrag im Sinne des ökonomischen Tauschs gewinnt an Bedeutung. Zugleich stellen Arbeitnehmer zunehmend Ansprüche an Selbstbestimmung und -verwirklichung in der Arbeit. Unternehmen richten ihrerseits an die Arbeitnehmer Anforderungen, ihre Arbeit selbstorganisiert, selbstverantwortlich und „unternehmerisch“, aber natürlich orientiert an den Erwartungen und Steuerungsvorgaben der Unternehmen, zu leisten. Ergebnisorientierung tritt im Leistungsverständnis zunehmend an die Stelle der Aufwandsund Arbeitszeitorientierung, wobei als Ergebnis immer häufiger der ökonomische Erfolg verstanden wird. Zugleich gewinnt die Arbeit auch vor dem Hintergrund der Massenarbeitslosigkeit und der Zunahme prekärer Arbeitsverhältnisse den Charakter eines erstrebenswerten Gutes. Institutionalisierte Formen der Anerkennung der Arbeitenden erodieren, der Kampf um Anerkennung wird individueller geführt.

Mit der Individualisierung der Arbeitsund Anerkennungsverhältnisse verliert das bisherige Arbeitsrecht an Reichweite und
Angemessenheit. Die Geltung tarifvertraglicher Mindeststandards wird infrage gestellt. Durch neue Formen der Selbstständigkeit innerhalb und außerhalb eines Arbeitsvertrags wird die Typisierung des Arbeitnehmerbegriffs als Basis für Schutzbedürftigkeit unterspült. Die Umstellung von Ausschluss- auf Zugangsentscheidungen unterminiert die Legitimationsmechanismen organisationaler In- und Exklusion und die Geltung arbeitsrechtlicher Normen. Schutznormen entsprechen nicht mehr ohne Weiteres den Anerkennungserwartungen von Beschäftigten, die Autonomieansprüche an ihre Arbeit stellen. Mit einem Selbst- und Fremdbild als Unternehmer seiner selbst verträgt sich die arbeitsrechtliche Typisierung als Schutzbefohlener nur schwer.

Das Arbeitsrecht folgt aufgrund seiner Eigenlogik und relativen Autonomie bislang nur begrenzt den Veränderungen. Dadurch kann es nach wie vor den Erosionsprozess der Anerkennungsordnung begrenzen. Das ist Chance und Gefahr zugleich: Chance, weil ohne arbeitsrechtliche Regulierung sich ein Prozess „vom Status zum Vertrag" durchsetzen würde, der die Anerkennungsbeziehungen im Betrieb fragil und hochdifferenziert - in Abhängigkeit von Nutzen und Marktposition der Beteiligten - werden ließe. Gefahr deshalb, weil zunehmend Beschäftigte und Arbeitssituationen aus dem Geltungsbereich des Arbeitsrechts herausfallen, was die Segmentierung der Arbeitsbeziehungen verstärken wird. Reformbedarf ist also gegeben, aber das Arbeitsrecht ist nach wie vor nötig: als Grenze einer Vermarktlichung der Arbeitsbeziehungen - und damit auch einer Individualisierung und Fragilität der Anerkennungsverhältnisse.
6 Der Vorschlag, an die Stelle des Status' des Arbeitnehmers, bezogen auf Beruf und Unternehmen, einen „Persönlichen Berufsstatus" zu setzen, entkoppelt vom Mitgliedschaftsstatus beim spezifischen beschäftigenden Unternehmen (Supiot 2000, S. 297ff.), ist zwar bedenkenswert, enthält aber die Gefahr, damit das „industrial citizenship" aus der Organisation herauszuverlagern. 
Annuß, G. (2004): Der Arbeitsvertrag als Grundlage des Arbeitsverhältnisses, in: Zeitschrift für Arbeitsrecht 35, S. 283-310

Baethge, M. (1991): Arbeit, Vergesellschaftung, Identität - Zur zunehmenden normativen Subjektivierung der Arbeit, in: Soziale Welt 1 , S. 6-20

Bender, G. (1997): Lohnarbeit zwischen Autonomie und Zwang, Frankfurt a. M./New York

Blanke, T. (2004): Thesen zur Zukunft des Arbeitsrechts, in: Kritische Justiz 37, S. 2-20

Boltanski, L./Chiapello, È. (2003): Der neue Geist des Kapitalismus, Konstanz

Castel, R. (2000): Die Metamorphosen der sozialen Frage, Konstanz Gerlmaier, A./Kastner, M. (2003): Was sind Neue Selbstständige?, in: Kastner, M. (Hrsg.): Neue Selbstständigkeit in Organisationen, München/Mering, S. 49-75

Habermas , J. (1988): Theorie des kommunikativen Handelns, Bd. 2, Frankfurt a. M.

Höland, A./Reim, U./Brecht, H. (2000): Flächentarifvertrag und Günstigkeitsprinzip, ZERP-Diskussionspapier 1

Holtgrewe, U./Voswinkel, S./Wagner, G. (Hrsg.) (2000): Anerkennung und Arbeit, Konstanz

Honneth, A. (1994): Kampf um Anerkennung. Zur moralischen Grammatik sozialer Konflikte, Frankfurt a. $M$.

Kocyba, H. (2000): Der Preis der Anerkennung: Von der tayloristischen Missachtung zur strategischen Instrumentalisierung der Subjektivität der Arbeitenden, in: Holtgrewe, U./Voswinkel, S./Wagner, G. (Hrsg.): Anerkennung und Arbeit, Konstanz, S. 127-140

Kocyba, H./Voswinkel, S. (2007): Krankheitsverleugnung - Das Janusgesicht sinkender Fehlzeiten, in: WSI-Mitteilungen 3, S. 131-137 Kotthoff, H. (1994): Betriebsräte und Bürgerstatus. Wandel und Kontinuität betrieblicher Mitbestimmung, München/Mering

Kotthoff, H. (2000): Anerkennung und sozialer Austausch, in: Holtgrewe, U./Voswinkel, S./Wagner, G. (Hrsg.): Anerkennung und Arbeit, Konstanz, S. 27-36

Leisering, L. (1998): Sozialstaat und Individualisierung, in: Friedrichs, J. (Hrsg.): Die Individualisierungs-These, Opladen, S. 65-78

Luhmann, N. (1981): Die Funktion des Rechts: Erwartungssicherung oder Verhaltenssteuerung?, in: Ders.: Ausdifferenzierung des Rechts, Frankfurt a. M., S. 73-91

Maine, H. S. (1997): Das alte Recht - sein Zusammenhang mit der Frühgeschichte der Gesellschaft und sein Verhältnis zu modernen Ideen, Baden-Baden

Marshall, T. H. (1992/1950): Bürgerrechte und soziale Klassen, Frankfurt a. M./New York

Moldaschl, M./Sauer, D. (2000): Internalisierung des Marktes - Zur neuen Dialektik von Kooperation und Herrschaft, in: Minssen, H. (Hrsg.): Begrenzte Entgrenzungen, Berlin, S. 205-224

Moldaschl, M./ Voß, G. G. (Hrsg.) (2002): Subjektivierung von Arbeit, München/Mering
Mückenberger, U./Supiot, A. (2000): Ordre public social und Gemeinschaft: Zwei Kulturen des Arbeitsrechts, in: Wagner, P./Didry, C./Zimmermann, B. (Hrsg.): Arbeit und Nationalstaat, Frankfurt a. M./New York, S. $100-127$

Peters, K. (2003): Individuelle Autonomie von abhängig Beschäftigten. Selbsttäuschung und Selbstverständigung unter den Bedingungen indirekter Unternehmenssteuerung, in: Kastner, M. (Hrsg.): Neue Selbstständigkeit in Organisationen, München/Mering, S. 77-106

Picker, E. (2005): Das Arbeitsrecht zwischen Marktgesetz und Machtansprüchen, in: Zeitschrift für Arbeitsrecht 36, S. 353-390

Pongratz, H. J./Voß, G. G. (2003): Arbeitskraftunternehmer, Berlin Schaub, G. (2005): Arbeitsrechts-Handbuch, München

Schmid, G. (2002): Wege in eine neue Vollbeschäftigung, Frankfurt a. M./ New York.

Schumann, M. (1998): Frißt die Shareholder-Value-Ökonomie die Modernisierung der Arbeit?, in: Hirsch-Kreinsen, H./Wolf, H. (Hrsg.): Arbeit, Gesellschaft, Kritik, Berlin, S. 19-30

Schwarze, R. (2005): Arbeitnehmerbegriff und Vertragstheorie. Der paternalistische Kern des Arbeitnehmerschutzes, in: Zeitschrift für Arbeitsrecht 36, S. 81-107

Simitis, S. (1984): Zur Verrechtlichung der Arbeitsbeziehungen, in: Kübler, F. (Hrsg.): Verrechtlichung von Wirtschaft, Arbeit und sozialer Solidarität, Frankfurt a. M., S. 73-165

Streeck, W. (1988): Status und Vertrag als Grundkategorien einer soziologischen Theorie der industriellen Beziehungen, WZB-discussion paper FS I 88-3, Berlin

Supiot, A. (2000): Wandel der Arbeit und Zukunft des Arbeitsrechts in Europa, in: Kocka, J./Offe, C. (Hrsg.): Geschichte und Zukunft der Arbeit, Frankfurt a. M./New York, S. 293-307

Supiot, A. (2001): Beyond Employment. Changes in Work and the Future of Labour Law in Europe, Oxford/New York

Trittin, W. (2003): Arbeitsvertrag im Wandel: Das Arbeitsergebnis ersetzt die Arbeitszeit, in: Kastner, M. (Hrsg.): Neue Selbstständigkeit in Organisationen, München/Mering, S. 139-159

Voswinkel, S. (2001): Anerkennung und Reputation - Die Dramaturgie industrieller Beziehungen, Konstanz

Voswinkel, S. (2002): Bewunderung ohne Würdigung? Paradoxien der Anerkennung doppelt subjektivierter Arbeit, in: Honneth, A. (Hrsg.): Befreiung aus der Mündigkeit. Paradoxien des gegenwärtigen Kapitalismus, Frankfurt a. M., S. 65-92

Voswinkel, S. (2003): Leistung und Anerkennung - sind Zielvereinbarungen eine Lösung?, in: Hangebrauck, U.-M./Kock, K./Kutzner, E./Muesmann, G. (Hrsg.): Handbuch Betriebsklima, München/Mering, S. 179196

Voswinkel, S. (2005): Reziprozität und Anerkennung in Arbeitsbeziehungen, in: Adloff, F./Mau, S. (Hrsg.): Vom Geben und Nehmen. Zur Soziologie der Reziprozität, Frankfurt a. M./New York, S. 237-256 Wagner, G. (2004): Anerkennung und Individualisierung, Konstanz 